\title{
A Genotype-Based Approach to Assessing the Association between Single Nucleotide Polymorphisms
}

\author{
Stefan Wellek Andreas Ziegler \\ aDivision of Biostatistics, Central Institute of Mental Health, Mannheim/University of Heidelberg, Mannheim, and \\ bInstitute of Medical Biometry and Statistics, University at Lübeck, Lübeck, Germany
}

\section{Key Words}

Confidence interval $\cdot$ Counting variable $\cdot \delta$-Method $\cdot$

Fisher's z-transformation • Lewontin's $D^{\prime} \cdot$ Linkage

disequilibrium $\cdot$ Pearson's $\rho$

\begin{abstract}
Measuring the extent of linkage disequilibrium (LD) between single nucleotide polymorphisms (SNPs) is of considerable importance, and many different between SNP association measures including Lewontin's $D^{\prime}$ and Pearson's correlation coefficient $\rho$ have been proposed. The vast majority of these association measures are based on haplotypes instead of genotypes. If no family data are available, the required additional haplotype estimation step is based on the assumption of Hardy-Weinberg equilibrium (HWE). In this paper we propose to estimate the extent of LD by using a genotype- rather than haplotype-based measure. Furthermore, we require of an appropriate measure of LD that it should remain invariant under the transition from haplotypes to diploid genotypes if HWE holds. We show that Pearson's $\rho$ fulfills this invariance property in contrast to a variety of different LD measures including $D^{\prime}$. We derive the asymptotic distribution of the empirical product-moment correlation $R$ for counting variables and construct asymptotically valid confidence intervals using Fisher's $z$-transformation. We demonstrate the validity of our approach by a numerical study of the coverage properties. We show that the loss in precision encountered by using genotype rather than hap-
\end{abstract}

lotype data for estimating the association between SNPs is negligible for practical purposes. We finally illustrate our approach with data from an association study of IL-4 associated phenotypes and polymorphisms from the human IL-4 receptor alpha chain gene (IL4R).

Copyright $\odot 2008$ S. Karger AG, Basel

\section{Introduction}

Single nucleotide polymorphisms (SNPs) are the standard markers in current genetic epidemiological research. They are available in a dense genome-wide map, can be typed highly automatically and are available in different chip technologies with hundreds of thousands of SNPs per chip. They have therefore been heralded as the catalyst to unravel the genetic basis of complex diseases [1], and several successful applications to common genetic diseases have been published very recently [2-5].

Genetic epidemiological studies utilizing SNPs rely on a certain degree of association between the markers, traditionally termed linkage disequilibrium (LD) in genetics. The strength of this association reflects the extent to which alleles at different SNPs are inherited together forming thus 'haplotypes' by descent from single ancestral chromosomes. For practical purposes, the association between SNPs is of considerable importance in a number of situations: First, when a causative variant is not assayed directly - which is likely for today's SNP 
chips -, the success of a study depends on the association between the functional variant and the closely neighboring SNP $[6,7]$. Second, for fine mapping studies, data on the association between pairs of SNPs may also be used to reduce the number of SNPs needed to be genotyped in a specific genetic region because many of these will provide redundant information [8]. Third, contrasting LD patterns between cases and controls can be used to test SNP disease associations $[9,10]$. Finally, with sufficient association between SNPs it is possible to reconstruct the evolution of the genome [11, 12].

Therefore, there is a clear need for measures of association between SNPs, and in past years many different statistics have been proposed [for an overview see, e.g., refs. 1, 13; for critical comparisons between different measures see 14-16]. The most important association measures are Lewontin's $D^{\prime}$ [17] and Pearson's productmoment correlation coefficient [6, 18-20]. In most current applications of these measures, the aim is to assess intra-gametic association between alleles of pairs of SNPs which requires knowledge of haplotypes. In case-control or cohort studies of unrelated subjects, however, only single SNP-wise genotypes are usually observed so that missing haplotype information must be reconstructed by means of some estimation procedure. This approach has several obvious shortcomings, among which reliance on the assumption of Hardy-Weinberg equilibrium (HWE) is the most important one in the present context.

In this paper, we adopt the general perspective of [20] proposing to replace the traditional intra-gametic (haplotype-based) measures by genotype-based measures of LD which allows dispensing with the haplotype estimation step. Furthermore, we require of an appropriate measure of LD that it should remain invariant under the transition from haplotypes to diploid genotypes if HWE holds. In other words, if HWE can be taken for granted - as has to be assumed for most statistical approaches to haplotyping -, the measure should not change its value when computed from genotype rather than haplotype frequencies. If this holds true, then another natural requirement refers to the loss in statistical precision encountered by using genotype rather than haplotype data for estimating the association between SNPs which should be moderate whenever the underlying populations are in HWE.

In the next section, we check Pearson's $\rho$ for invariance under exchanging haplotype with genotype information, and review a number of other basic properties usually required in statistics of a reasonable measure of association. It turns out that $\rho$ satisfies all these requirements - in contrast to Lewontin's $D^{\prime}$, and we therefore proceed by investigating the asymptotic distribution of the empirical product-moment correlation coefficient as computed with genotype frequency data. In doing so, we exploit the fact that $\hat{\rho}=R$ can be represented as a simple continuously differentiable function of the population genotype frequencies making up the joint distribution of any pair of random variables taking values in the set $\{0,1,2\}$. Because the corresponding observed frequencies are consistent and asymptotically normal estimators of these parameters, it follows that $R$ has an asymptotic normal distribution, and its variance admits consistent estimation. Our result generalizes the findings of [20] who discussed the asymptotic distribution of the empirical productmoment correlation between numbers of alleles under the null hypothesis of independence only. With the extension, we are able to calculate asymptotically valid confidence intervals for $\rho$ in a straightforward way, and we describe the details of an interval estimation procedure using Fisher's $z$ transformation as pivot. Subsequently, we study the coverage properties of the respective asymptotic confidence intervals by exact numerical computation for sample sizes less than 30 and by Monte-Carlo simulation otherwise. The practical use of the estimation procedure in the genetic epidemiology of complex diseases is illustrated by means of a data set taken from a study of the association between IL- 4 associated phenotypes and polymorphisms from the human IL-4 receptor alpha chain gene (IL4R). Finally, we consider the possibility of improving the efficiency of the estimator of the correlation between diallelic genetic markers by analyzing haplotype rather than genotype distributions whenever the underlying population conforms to Hardy-Weinberg equilibrium (HWE). The question whether applying other common association measures like Kendall's Tau, Spearman's Rho, or the polychoric correlation to genotype data can be considered a real alternative to the approach based on the product-moment correlation, and this is addressed in the Discussion.

\section{Materials and Methods}

Properties of the Product-Moment Correlation as a Measure of Association between Loci

Let us assume that the analysis focuses on a specific couple of SNPs which are in close vicinity on the same chromosome and that the corresponding population of haplotype probabilities is given by the entries in the $2 \times 2$ contingency table 1 . Measuring the association between these two loci at the haplotype level by means of Pearson's $\rho$ fits in the more general framework intro- 
Table 1. Distribution of haplotypes formed by a generic couple of SNPs. Alleles at locus 1 are $a$ and $A$, alleles at locus 2 are $b$ and $B$

\begin{tabular}{lll}
\hline Locus 1 & Locus 2 & \\
\cline { 2 - 3 } & $b$ & $B$ \\
\hline$a$ & $h_{1}$ & $h_{2}$ \\
$A$ & $h_{3}$ & $h_{4}$ \\
\hline
\end{tabular}

duced in the next section where $\rho$ and its sample analogue, denoted by $R$, are studied for the case of two counting variables taking values in the set $\{0, \ldots, K\}$ where $K$ stands for a given integer $\geq 2$. Under the additional assumption that the population of individuals from which the bivariate sample $\left(X_{\nu}, Y_{\nu}\right)_{1 \leq \nu \leq n}$ of allele counts across the diploid set is taken, strictly conforms to HardyWeinberg equilibrium (HWE), the joint distribution of genotypes defined in terms of the same pair of SNPs is given by the entries in the $3 \times 3$ contingency table 2 . Because HWE implies that the joint genotype distribution is uniquely determined by the haplotype frequencies, it is reasonable to require then that the result of measuring the association between the two SNP loci under consideration does not depend on whether we look at two-locus haplotypes or diploid genotypes from two SNPs. In other words, the preferred measure of association should remain invariant under switching over to computing it from the genotype frequencies of table 2 instead of the haplotype frequencies of table 1.

In order to verify that the population product-moment correlation coefficient will never change its value when computed from table 2 instead of table 1, we first recall that in the binary case, $\rho$ is given by [see, e.g., ref. 13, table 2]:

$$
\rho=\frac{h_{1} h_{4}-h_{2} h_{3}}{\sqrt{\left(h_{1}+h_{2}\right)\left(h_{1}+h_{3}\right)\left(h_{2}+h_{4}\right)\left(h_{3}+h_{4}\right)}} .
$$

On the other hand, if the joint distribution of $(X, Y)$ is as shown in table 2, it admits the representation

$$
(X, Y)=\left(A_{1}+A_{2}, B_{1}+B_{2}\right),
$$

where $A_{1}$ and $B_{1}\left[A_{2}\right.$ and $\left.B_{2}\right]$ count the number of $A$ and $B$ alleles on the first [second] chromosome, respectively. Under the assumption of HWE, the random pairs $\left(A_{1}, B_{1}\right),\left(A_{2}, B_{2}\right)$ are independent of each other and have the same distribution, and this implies

$$
\begin{aligned}
& \operatorname{Var}(X)=\operatorname{Var}\left(A_{1}\right)+\operatorname{Var}\left(A_{2}\right)=2\left(h_{1}+h_{2}\right)\left(h_{3}+h_{4}\right), \\
& \operatorname{Var}(Y)=\operatorname{Var}\left(B_{1}\right)+\operatorname{Var}\left(B_{2}\right)=2\left(h_{1}+h_{3}\right)\left(h_{2}+h_{4}\right) .
\end{aligned}
$$

Similarly, it follows that

$$
\operatorname{Cov}(X, Y)=\operatorname{Cov}\left(A_{1}, B_{1}\right)+\operatorname{Cov}\left(A_{2}, B_{2}\right)=2\left(h_{1} h_{4}-h_{2} h_{3}\right) .
$$

Hence, the genotype-based product-moment correlation between the two loci is given by

$$
\operatorname{Corr}(X, Y)=\frac{2\left(h_{1} h_{4}-h_{2} h_{3}\right)}{\sqrt{4\left(h_{1}+h_{2}\right)\left(h_{3}+h_{4}\right)\left(h_{1}+h_{3}\right)\left(h_{2}+h_{4}\right)}}
$$

and thus identical to (1).
Table 2. Joint genotype distribution obtained from the haplotype frequencies shown in table 1 under Hardy-Weinberg equilibrium with, respectively, $X$ and $Y$ denoting the number of $A$-alleles at SNP 1 and $B$-alleles at SNP 2

\begin{tabular}{llll}
\hline$X$ & $Y$ & \\
\cline { 2 - 3 } & 0 & 1 & 2 \\
\hline 0 & $h_{1}^{2}$ & $2 h_{1} h_{2}$ & $h_{2}^{2}$ \\
1 & $2 h_{1} h_{3}$ & $2\left(h_{1} h_{4}+h_{2} h_{3}\right)$ & $2 h_{2} h_{4}$ \\
2 & $h_{3}^{2}$ & $2 h_{3} h_{4}$ & $h_{4}^{2}$ \\
\hline
\end{tabular}

In order to demonstrate that under HWE, the derived equality of the genotype-based product-moment correlation and the haplotype-based product-moment correlation is still approximately valid when both measures are computed with observed rather than population genotype frequencies, we performed a small Monte-Carlo simulation study. The results are displayed in table 4 , and it can be concluded that a sample size of $n=100$ suffices to ensure that the expected absolute difference between the haplotype- and the genotype-based estimate of $\rho$ becomes as small as about 0.02 . If $n$ is in the order of current genome-wide association studies [5], the expected discrepancy decreases to values less than 0.006 .

If $\mathrm{HWE}$ is violated as is the case in the third of the scenarios specified in table 3, the haplotype-based estimator is strongly biased and shows marked discrepancies from the genotype-based estimator. Its bias persists even when the sample size is increased up to 1,600 . In contrast, the genotype-based estimator still is approximately unbiased even for sample sizes as small as 50 .

In addition to satisfying the basic invariance condition which other measures fail to meet, the product-moment correlation between SNPs, or even more generally counting variables $X$ and $Y$ taking values in $\{0,1, \ldots, K\}-$ for SNPs $K \equiv 2$ - has a number of further desirable properties. In particular, the following statements hold true whenever both marginal distributions are nondegenerate, where non-degenerate means that they are not concentrated on a subset of the common range $\{0,1, \ldots, K\}$ :

(i) $X$ independent of $Y \Rightarrow \rho=0$;

(ii) $\rho=1 \Leftrightarrow P(X=Y)=1$;

(iii) $\rho=-1 \Leftrightarrow P(X=K-Y)=1$.

All these arguments make the product-moment correlation $\rho$ well suited for assessing the degree of association between SNPs, irrespective of whether their distribution is in HWE or deviates from HWE.

Lewontin's $D^{\prime}$ as a Measure of Association between Loci

Although properties (ii) and (iii) seem to be essential for any reasonable measure of association, they do not hold for Lewontin's $D^{\prime}$, despite the popularity of the latter in the literature from genetic epidemiology [see, e.g., ref. 1, ch. 9]. Actually, as a measure of LD for haplotypes involving two diallelic loci, $D^{\prime}$ is well known [see ref. 21, pp. 256-257] to indicate 'perfect association' (i.e., to take on value 1) whenever a single entry in table 1 is zero, irrespective of the amount of probability mass assigned to the other di- 
Table 3. Parameter constellation for three scenarios checked for discrepancies between the haplotype-based and the genotype-based estimators of the product-moment correlation $\rho$ between the allele counts $X$ and $Y$ at two loci

\begin{tabular}{llllllllllll}
\hline Scenario & $\pi_{00}$ & $\pi_{01}$ & $\pi_{02}$ & $\pi_{10}$ & $\pi_{11}$ & $\pi_{12}$ & $\pi_{20}$ & $\pi_{21}$ & $\pi_{22}$ & $\rho$ & HWE \\
\hline 1 & 0.2025 & 0.0900 & 0.0100 & 0.0000 & 0.4050 & 0.0900 & 0.0000 & 0.0000 & 0.2025 & 0.8182 & yes \\
2 & 0.0100 & 0.0200 & 0.0100 & 0.0400 & 0.1600 & 0.1200 & 0.0400 & 0.2400 & 0.3600 & 0.2182 & yes \\
3 & 0.0900 & 0.0050 & 0.0050 & 0.1200 & 0.0400 & 0.0400 & 0.0350 & 0.0350 & 0.6300 & 0.7420 & no \\
\hline
\end{tabular}

HWE = Hardy-Weinberg equilibrium; $\pi_{i j}=P\left(X_{\nu}=i, Y_{\nu}=j\right), 0 \leq i, j \leq 2$ are the population genotype frequencies.

agonal of the table. A simple numerical example is given if we have $h_{3}=0, h_{1}=h_{4}=1 / 4, h_{2}=1 / 2$.

$D^{\prime}$ also fails to remain invariant under replacing haplotype with HWE-consistent genotype frequencies. This is shown by the following counterexample: As before, let the haplotype distribution be given by $\left(h_{1}, h_{2}, h_{3}, h_{4}\right)=(1 / 4,1 / 2,0,1 / 4)$. Under HWE, the corresponding joint distribution of genotypes is given by the $3 \times 3$-matrix

$$
\left(\begin{array}{ccc}
\frac{1}{16} & \frac{1}{4} & \frac{1}{4} \\
0 & \frac{1}{8} & \frac{1}{4} \\
0 & 0 & \frac{1}{16}
\end{array}\right)
$$

Applying the definition of $D^{\prime}$ for the multiallelic case [ref. 21, p. 237] to these numbers yields a $D^{\prime}$ value as low as 0.3469 although we have $D^{\prime}=1$ in the underlying distribution of haplotypes. By these reasons, $D^{\prime}$ is not considered a serious competitor to the classical Pearson product moment correlation measure. The first of these objections also applies to the association probability $\eta$ [see, e.g., ref. 1] which coincides with Lewontin's $D^{\prime}$ whenever there holds $h_{1} h_{4}>h_{2} h_{3}$ and $\left(h_{3}+h_{4}\right) \leq\left(h_{2}+h_{4}\right)$.

\section{Asymptotic Distribution of the Empirical Product-Moment}

Correlation $\boldsymbol{R}$ between Counting Variables

In this section we study the distribution of $R$ when computed from a sample of $n$ mutually independent random pairs $\left(X_{1}\right.$, $\left.Y_{1}\right), \ldots,\left(X_{n}, Y_{n}\right)$ such that for each $\nu=1 \ldots, n$, both $X_{\nu}$ and $Y_{\nu}$ take integer values between 0 and $K$, inclusive. The joint distribution of each such pair is given by $P\left(X_{\nu}=i, Y_{\nu}=j\right)=\pi_{i j}, 0 \leq i, j \leq K$, where the $\pi_{i j}$ are the basic population parameters. Referring to haplotype and genotype data, $K=1$ and $K=2$, respectively. Likewise, $\pi_{i j}$ is used in the sequel as a generic symbol whose concrete meaning depends on the context. If $\rho$ is to be estimated from haplotype frequency data, then we have $\pi_{00}=h_{1}, \ldots, \pi_{11}=h 4$ (see table 1). Dealing with the joint distribution of genotype-wise allele counts at two different SNPs, then without the restriction of assuming HWE, the $\pi_{i j}$ stand for the populations frequencies of the 9 combinations of numbers of $A$ and $B$ alleles and denote arbitrary non-negative numbers summing up to unity.

The natural starting point for studying the distribution of $R$ in both settings, is to represent it as a function of the relative frequencies $\hat{\pi}_{i j}=N_{i j} / n$ estimating the parameters of the correspond- ing multinomial distribution. In any case, the population correlation coefficient between counting variables can be written

$$
\rho(\boldsymbol{\pi})=\frac{c(\boldsymbol{\pi})}{\sqrt{v_{1}(\boldsymbol{\pi}) v_{2}(\boldsymbol{\pi})}},
$$

where

$$
\begin{aligned}
c(\boldsymbol{\pi}) & =\sum_{i=0}^{K} \sum_{j=0}^{K} i j \pi_{i j}-e_{1}(\boldsymbol{\pi}) e_{2}(\boldsymbol{\pi}), & & \\
v_{1}(\boldsymbol{\pi}) & =\sum_{i=0}^{K} i^{2} \pi_{i+}-e_{1}^{2}(\boldsymbol{\pi}), & v_{2}(\boldsymbol{\pi}) & =\sum_{j=0}^{K} j^{2} \pi_{+j}-e_{2}^{2}(\boldsymbol{\pi}), \\
e_{1}(\boldsymbol{\pi}) & =\sum_{i=0}^{K} i \pi_{i+}, & e_{2}(\boldsymbol{\pi}) & =\sum_{j=0}^{K} j \pi_{+j}, \\
\pi_{i+} & =\sum_{j=0}^{K} \pi_{i j}, i=0, \ldots, K, & \pi_{+j} & =\sum_{i=0}^{K} \pi_{i j}, j=0, \ldots, K .
\end{aligned}
$$

Clearly, the sample correlation coefficient $R$ is just the plug-in estimator of $\rho(\boldsymbol{\pi})$ to be computed by substituting each $\pi_{i j}$ by the homologous observed proportion $\hat{\pi}_{i j}=N_{i j} / n$ at every place where it appears in the above formulae. Because $\rho$ is a continuously differentiable function of $\pi_{i j}$, the $\delta$-method may be employed for deriving the asymptotic distribution of $R=\rho(\hat{\boldsymbol{\pi}})$ [ref. 22, ch. 14]. This widely used mathematical result allows us to conclude that under any $\boldsymbol{\pi}$ for which $\rho(\boldsymbol{\pi})$ is a well-defined real number, $R$ is asymptotically normal with expectation $\rho(\boldsymbol{\pi})$ and asymptotic variance which is shown in Appendix I to be given by

$$
\begin{aligned}
\operatorname{Var}_{a s}(R)=n^{-1} & \left\{\sum _ { i = 0 } ^ { K } \sum _ { j = 0 } ^ { K } \left[\frac{i j-i e_{2}(\boldsymbol{\pi})-j e_{1}(\boldsymbol{\pi})}{v_{2}^{1 / 2}(\boldsymbol{\pi}) v_{2}^{1 / 2}(\boldsymbol{\pi})}\right.\right. \\
& \left.-\frac{1}{2} \rho(\boldsymbol{\pi})\left(\frac{i\left(i-2 e_{1}(\boldsymbol{\pi})\right)}{v_{1}(\boldsymbol{\pi})}+\frac{j\left(j-2 e_{2}(\boldsymbol{\pi})\right)}{v_{2}(\boldsymbol{\pi})}\right)\right]^{2} \pi_{i j} \\
& -\left[\sum _ { i = 0 } ^ { K } \sum _ { j = 0 } ^ { K } \left(\frac{i j-i e_{2}(\boldsymbol{\pi})-j e_{1}(\boldsymbol{\pi})}{v_{1}^{1 / 2}(\boldsymbol{\pi}) v_{2}^{1 / 2}(\boldsymbol{\pi})}\right.\right. \\
& \left.\left.\left.-\frac{1}{2} \rho(\boldsymbol{\pi})\left(\frac{i\left(i-2 e_{1}(\boldsymbol{\pi})\right)}{v_{1}(\boldsymbol{\pi})}+\frac{j\left(j-2 e_{2}(\boldsymbol{\pi})\right)}{v_{2}(\boldsymbol{\pi})}\right)\right) \pi_{i j}\right]^{2}\right\} .
\end{aligned}
$$

Since the expression on the right-hand side of (5) is another continuously differentiable function of the $\pi_{i j}$ and the latter are consistently estimated by the $\hat{\pi}_{i j}$, a consistent estimator of the asymptotic variance of $R$ is obtained by replacing each multinomial parameter with the corresponding proportion observed in the 
Table 4 Finite sample behavior of the haplotype- as compared to the genotype-based estimator of the productmoment correlation $\rho$ between $X$ and $Y$ for the scenarios of table 3

\begin{tabular}{lrlll}
\hline Scenario & $n$ & $\hat{\rho}_{\text {HAP }}$ & $\hat{\rho}_{\text {GENO }}$ & $\left|\hat{\rho}_{\text {HAP }}-\hat{\rho}_{\text {GENO }}\right|$ \\
\hline 1 & 50 & $0.81787 \pm 0.04975$ & $0.81654 \pm 0.06106$ & $0.02668 \pm 0.02326$ \\
& 100 & $0.81791 \pm 0.03527$ & $0.81708 \pm 0.04279$ & $0.00083 \pm 0.02394$ \\
& 400 & $0.81804 \pm 0.01754$ & $0.81798 \pm 0.02118$ & $0.00935 \pm 0.00718$ \\
& 800 & $0.81821 \pm 0.01234$ & $0.81807 \pm 0.00149$ & $0.00653 \pm 0.00501$ \\
& 1,600 & $0.81821 \pm 0.00880$ & $0.81818 \pm 0.01059$ & $0.00467 \pm 0.00356$ \\
\hline 5 & 50 & $0.21350 \pm 0.14318$ & $0.22146 \pm 0.14411$ & $0.03019 \pm 0.02774$ \\
& 100 & $0.21647 \pm 0.08722$ & $0.21810 \pm 0.10122$ & $0.02147 \pm 0.01829$ \\
& 400 & $0.21676 \pm 0.04872$ & $0.21755 \pm 0.05077$ & $0.01130 \pm 0.00881$ \\
& 800 & $0.21721 \pm 0.03435$ & $0.21797 \pm 0.03582$ & $0.00803 \pm 0.00615$ \\
& 1,600 & $0.21740 \pm 0.02428$ & $0.21810 \pm 0.02527$ & $0.00578 \pm 0.00439$ \\
\hline 50 & $0.63394 \pm 0.08944$ & $0.74274 \pm 0.08963$ & $0.10738 \pm 0.03717$ \\
& 100 & $0.63515 \pm 0.06212$ & $0.74162 \pm 0.06234$ & $0.10645 \pm 0.02567$ \\
& 400 & $0.63623 \pm 0.03067$ & $0.74191 \pm 0.03075$ & $0.10568 \pm 0.01255$ \\
& 800 & $0.63623 \pm 0.02168$ & $0.74171 \pm 0.02166$ & $0.10549 \pm 0.00885$ \\
& 1,600 & $0.63659 \pm 0.01538$ & $0.74192 \pm 0.01543$ & $0.10533 \pm 0.00624$
\end{tabular}

Simulation results based on 40,000 replications of each Monte Carlo experiment. Reported are mean \pm standard deviation of the empirical estimators. $\hat{\rho}_{H A P}$, haplotype-based estimator of the product-moment correlation coefficient; $\hat{\rho}_{G E N O}$, genotype-based estimator of the product-moment correlation coefficient.

sample. Denoting the resulting empirical counterpart of $\operatorname{Var}_{a s}(R)$ by $\widehat{\operatorname{Var}}_{a s}(R)$, asymptotic inference about $\rho(\boldsymbol{\pi})$ can be performed through treating $R$ as a variable with distribution $N(\rho(\pi)$, $\left.\widehat{\operatorname{Var}}_{a s}(R)\right)$.

For the squared asymptotic standard error of $R$ a similar formula has been provided by Brown and Benedetti [23]. Although it admittedly looks somewhat lengthy, its mathematical structure is far from being complicated, and writing a source program implementing the computation of $\operatorname{Var}_{a s}(R)$ (thus also of $\widehat{\operatorname{Var}}_{a s}(R)$ ) is a simple exercise. It is important to note that the variance formula (5) for distributions of counting variables differs from the classical variance formula $\operatorname{Var}_{a s}(R)=n^{-1}\left(1-\rho^{2}\right)^{2}$ for bivariate normally distributed random variables $\left(X_{\nu}, Y_{\nu}\right)$. However, as is shown in Part II of the Appendix, the right-hand side of (5) reduces to $n^{-1}$ under the null hypothesis of independence of $X_{\nu}$ and $Y_{\nu}$. This is in accordance with the finding of ref. [24] that in the independence case, $R$ has asymptotic variance $n^{-1}$ for any underlying theoretical distribution with finite moments of order 4 at least. Note that in practice $n^{-1}$ is frequently replaced by $(n-3)^{-1}$ in the case of a bivariate normal distribution.

\section{Construction of Asymptotically Valid Confidence Intervals}

To avoid the common difficulties encountered in interval estimation of parameters ranging over a bounded space of possible values, it seems advisable to use Fisher's $z$ transformation $\rho \mapsto$ $z(\rho)=1 / 2 \log ((1+\rho) /(1-\rho))$ which maps the original parameter space onto the whole real line. Putting $Z=z(R), \zeta(\boldsymbol{\pi})=z(\rho(\boldsymbol{\pi}))$, it follows from another application of the $\delta$-method that $Z$ is asymptotically normal with expectation $\zeta(\boldsymbol{\pi})$ and variance $\operatorname{Var}_{a s}(Z)=$ $\left(1-\rho^{2}(\boldsymbol{\pi})\right)^{-2} \operatorname{Var}_{a s}(R)$. We stress that this $z$ transformation is no longer a variance-stabilizing transformation when $R$ is computed with data from nonnormal distributions because the right hand side of Equation (5) is not constant in $\rho$. $\operatorname{Var}_{a s}(Z)$ can be consistently estimated by $\widehat{\operatorname{Var}}_{a s}(Z)=\left(1-R^{2}\right)^{-2} \widehat{\operatorname{Var}}_{a s}(R)$.

Computation of a confidence interval of asymptotic level $1-\alpha$ for $\rho(\boldsymbol{\pi})$ can now be carried out in the usual way: In a first step, the limits of an approximate $1-\alpha$ confidence interval for $\zeta(\pi)$ are determined from

$$
\begin{aligned}
& \underline{\zeta}(1-\alpha)=Z-u_{1-\alpha / 2} \cdot \widehat{\operatorname{Var}}_{a s}^{1 / 2}(Z), \\
& \underline{\zeta}(1-\alpha)=Z+u_{1-\alpha / 2} \cdot \widehat{\operatorname{Var}}_{a s}^{1 / 2}(Z),
\end{aligned}
$$

with $u_{1-\alpha / 2}$ denoting the $(1-\alpha / 2)$ quantile the standard normal distribution. Applying the inverse of Fisher's $z$-transformation to these random quantities and using the analogous notation for the limits of an asymptotic $(1-\alpha)$ confidence interval for the parameter of interest gives

$$
\underline{\rho}(1-\alpha)=\tanh \underline{\zeta}(1-\alpha), \bar{\rho}(1-\alpha)=\tanh \bar{\zeta}(1-\alpha) .
$$

For covering the case that the observed correlation coefficient vanishes $-z(\cdot)$ obviously has a singularity at $0-$,

$$
\begin{aligned}
& \underline{\rho}(1-\alpha)=-u_{1-\alpha / 2} \cdot \widehat{\operatorname{Var}}_{a s}^{1 / 2}(R), \\
& \bar{\rho}(1-\alpha)=u_{1-\alpha / 2} \cdot \widehat{\operatorname{Var}}_{a s}^{1 / 2}(\mathrm{R}) \text { for for } \mathrm{R}=0 \text { we set. }
\end{aligned}
$$

The statement that this procedure yields a confidence interval of asymptotic level $1-\alpha$ for $\rho(\boldsymbol{\pi})$ holds true under the following straightforward conditions on the parameter $\pi$ identifying the distribution from which the $\left(X_{\nu}, Y_{\nu}\right)$ are taken:

$$
\begin{aligned}
& \text { (C1) } v_{1}(\boldsymbol{\pi})>0, v_{2}(\boldsymbol{\pi})>0 ; \\
& (\mathrm{C} 2)|\rho(\boldsymbol{\pi})|<1 .
\end{aligned}
$$


Although (C1) and (C2) imply that $v_{1}(\hat{\boldsymbol{\pi}}), v_{2}(\hat{\boldsymbol{\pi}})$ and $\rho(\hat{\boldsymbol{\pi}})$ almost surely converge to non-degenerate limits as $n \rightarrow \infty$, in finite samples exceptions can happen with positive probability. In order to extend the estimation procedure to cases where such exceptions occur, we propose the following conventions:

(D1) Whenever at least one of the two empirical variances $v_{1}(\hat{\boldsymbol{\pi}}), v_{2}(\hat{\boldsymbol{\pi}})$ vanishes, both the point estimator $\rho(\hat{\boldsymbol{\pi}})$ and the confidence limits $\rho(1-\alpha), \bar{\rho}(1-\alpha)$ are set equal to zero.

(D2a) When the joint empirical distribution of the $\left(X_{\nu}, Y_{\nu}\right)$ turns out to be concentrated on the main diagonal of the associated contingency table so that we find $\rho(\hat{\boldsymbol{\tau}})=1$, one of the observations from the cell with the largest absolute frequency is transferred to the neighboring off-diagonal cell. The lower confidence limit is determined from this slightly modified table in the same way as in the non-exceptional cases, leading to a pseudo-point estimate of $\rho(\hat{\boldsymbol{\pi}})$ within the domain of definition of the $z$-transformation. The upper confidence limit is set equal to 1 .

(D2b) An analogous modification to the primarily observed contingency table is performed in cases where we have $\sum_{k=0}^{K} \hat{\pi}_{k, K-k}=1$ implying that $\rho(\hat{\boldsymbol{\pi}})=-1$.

The rules for handling exceptional cases in which either $\rho(\hat{\boldsymbol{\pi}})$ or $\zeta(\hat{\boldsymbol{\pi}})$ fails to be well defined, are mainly of relevance for numerical studies on the coverage behavior of $(\rho(1-\alpha), \bar{\rho}(1-\alpha))$ in small samples from populations with large $|\overline{\rho(\pi)}|$. For the sake of completeness, they have also been implemented in a freely available $S A S$ macro. This program has been designed as a discrete variable analogue to the traditional $S A S$ procedure CORR. However, in contrast to PROC CORR, the focus is on confidence intervals rather than $\mathrm{p}$ values calculated under the null hypothesis of independence. Other computational tools of some value for the purpose of assessing the association between counting variables are the SAS procedure FREQ and the Pearson's Correlation Test procedure of StatXact. In fact, both programs compute the empirical standard error of $R$ by plugging in the observed frequencies of all possible values of $(X, Y)$ in a formula equivalent to (5). However, confidence limits are computed without making use of the $z$-transformation and applying suitable rules for handling cases where $|R|$ turns out to be unity. Whenever this happens, the FREQ procedure just sets both confidence limits equal to the point estimate, and the same approach is used by StatXact.

Doubling the Sample Size in Assessing Associations under Hardy-Weinberg Equilibrium?

We have shown that the haplotype-based and the genotypebased product-moment correlation coefficients coincide when HWE is met. It therefore seems natural to compare the efficiency of the genotype-based estimator compared with that of the haplotype-based estimator. Indeed, the sample size of the haplotypebased estimator is doubled but the question whether the efficiency of statistical inference about genetic markers being in HWE can be enhanced through relating to a sample of size $2 n$ from the distribution of alleles rather than the diploid genotypes of $n$ individuals has been raised and answered in the negative by Sasieni [26] for a case-control study involving a single marker. Of course, minimum requirements for recommending a haplotype- rather than a genotype-based analysis under HWE in the present context are (1) in the best case - phased rather than conventional unphased genotyping and successful verification of the HWE condition -, doubling of sample sizes entails substantial gain in efficiency of the confidence procedure,

Measuring Association between SNPs
(2) the availability of a test for establishing goodness rather than lack of fit of the joint distribution of two SNPs to the model specified in table 2 . Such a test might be obtained through generalizing the ideas behind the approaches used in ref. [27] for constructing tests for compatibility of an observed distribution of single-SNP genotypes with HWE.

Let us denote the asymptotic variance of the empirical product-moment correlation based on a sample of size $2 n$ from some given haplotype distribution (see table 1 ) and a sample of $n$ observations from the associated distribution of diploid genotypes (table 2) by $\operatorname{Var}_{a s}\left(\hat{\rho}_{2 n}^{(H)}\right)$ and $\operatorname{Var}_{a s}\left(\hat{\rho}_{n}^{(D)}\right)$, respectively. Then, defining the asymptotic efficiency of $\left.\hat{\rho}_{2 n}^{(H)}\right)$ relative to $\left(\hat{\rho}_{n}^{(D)}\right)$ in the usual way [see ref. $28, \S 10.1$ ] leads to considering

$$
e^{*}\left(\hat{\rho}_{n}^{(H)}, \hat{\rho}_{2 n}^{(D)}\right)=\operatorname{Var}_{a s}\left(\hat{\rho}_{n}^{(D)}\right) / \operatorname{Var}_{a s}\left(\hat{\rho}_{2 n}^{(H)}\right) .
$$

The expression on the right-hand of this equation is an algebraic function of the true haplotype frequencies $h_{1}, \ldots, h_{4}$ which is readily evaluated by means of formula (5).

\section{Results}

\section{Finite-Sample Properties of the Genotype-Based} Estimator

Table 5 reports major results of a numerical study of the distribution of $R$ and the coverage proportion of $(\underline{\rho}(1-\alpha), \bar{\rho}(1-\alpha))$ in finite samples from four different distributions concentrated on the set $\{0,1,2\} \times\{0,1,2\}$. Under the first of these configurations, $X_{\nu}$ and $Y_{\nu}$ are independent, under the others, the population correlation coefficient $\rho(\pi)$ ranges up to about 0.85 .

Comparison of the asymptotic variance $\operatorname{Var}_{a s}(R)$ to the exact variance $\sigma^{2}(R)$ shows that a sample size of $n=60$ suffices for reducing the approximation error to a practically negligible amount. Unfortunately, this does not automatically indicate satisfactory goodness of fit of the distribution of $R$ or $\mathrm{z}(R)$ as a whole to a normal distribution. As becomes evident from the last row of the third block of entries in table 5 (relating to $n=60$ ), even when the large-sample variance comes very close to the exact value of $\sigma^{2}(R)$, the coverage of the confidence interval computed by means of it can still be unsatisfactory. In order to ensure practically acceptable accuracy in terms of coverage proportion even under the most unfavorable configurations, three-figured sample sizes are required.

At first sight, the coverage probabilities obtained for the largest value of $\rho(\pi)$ look strange because the discrepancy between actual and nominal coverage proportion increases with the sample size up to $n=60$. This apparently counterintuitive phenomenon can, however, be explained easily. For the smallest of the sample sizes investigated here, the probability of obtaining an empirical 
Table 5. Finite sample results for pairs of variables taking on values in $\{0,1,2\}$

\begin{tabular}{|c|c|c|c|c|c|c|c|c|c|c|c|c|c|}
\hline$n$ & $\rho(\pi)$ & $\pi_{00}$ & $\pi_{01}$ & $\pi_{02}$ & $\pi_{10}$ & $\pi_{11}$ & $\pi_{12}$ & $\pi_{20}$ & $\pi_{21}$ & $\pi_{22}$ & $\sigma^{2}(R)$ & $\operatorname{Var}_{a s}(R)$ & $\operatorname{Prob}_{C}(\underline{\rho}, \bar{\rho})$ \\
\hline \multirow[t]{4}{*}{15} & 0.0000 & $1 / 9$ & $1 / 9$ & $1 / 9$ & $1 / 9$ & $1 / 9$ & $1 / 9$ & $1 / 9$ & $1 / 9$ & $1 / 9$ & 0.0708 & 0.0667 & 0.9085 \\
\hline & 0.1562 & $5 / 16$ & $5 / 24$ & $5 / 48$ & $3 / 32$ & $3 / 64$ & $3 / 64$ & $1 / 16$ & $1 / 16$ & $1 / 16$ & 0.0741 & 0.0681 & 0.8686 \\
\hline & 0.6250 & $1 / 4$ & $1 / 24$ & $1 / 24$ & $1 / 24$ & $1 / 4$ & $1 / 24$ & $1 / 24$ & $1 / 24$ & $1 / 4$ & 0.0497 & 0.0460 & 0.8385 \\
\hline & 0.8347 & $15 / 32$ & 0 & $1 / 32$ & $3 / 128$ & $45 / 128$ & 0 & $1 / 256$ & $1 / 256$ & $15 / 128$ & 0.0351 & 0.0335 & 0.9491 \\
\hline \multirow[t]{4}{*}{30} & 0.0000 & $1 / 9$ & $1 / 9$ & $1 / 9$ & $1 / 9$ & $1 / 9$ & $1 / 9$ & $1 / 9$ & $1 / 9$ & $1 / 9$ & 0.0344 & 0.0333 & 0.9365 \\
\hline & 0.1562 & $5 / 16$ & $5 / 24$ & $5 / 48$ & $3 / 32$ & $3 / 64$ & $1 / 16$ & $3 / 64$ & $1 / 16$ & $1 / 16$ & 0.0357 & 0.0340 & 0.9156 \\
\hline & 0.6250 & $1 / 4$ & $1 / 24$ & $1 / 24$ & $1 / 24$ & $1 / 4$ & $1 / 24$ & $1 / 24$ & $1 / 24$ & $1 / 4$ & 0.0237 & 0.0230 & 0.9131 \\
\hline & 0.8347 & $15 / 32$ & 0 & $1 / 32$ & $3 / 128$ & $45 / 128$ & 0 & $1 / 256$ & $1 / 256$ & $15 / 128$ & 0.0174 & 0.0168 & 0.9355 \\
\hline \multirow[t]{4}{*}{60} & 0.0000 & $1 / 9$ & $1 / 9$ & $1 / 9$ & $1 / 9$ & $1 / 9$ & $1 / 9$ & $1 / 9$ & $1 / 9$ & $1 / 9$ & 0.0168 & 0.0167 & 0.9457 \\
\hline & 0.1562 & $5 / 16$ & $5 / 24$ & $5 / 48$ & $3 / 32$ & $3 / 64$ & $3 / 64$ & $1 / 16$ & $1 / 16$ & $1 / 16$ & 0.0175 & 0.0170 & 0.9357 \\
\hline & 0.6250 & $1 / 4$ & $1 / 24$ & $1 / 24$ & $1 / 24$ & $1 / 4$ & $1 / 24$ & $1 / 24$ & $1 / 24$ & $1 / 4$ & 0.0116 & 0.0115 & 0.9419 \\
\hline & 0.8347 & $15 / 32$ & 0 & $1 / 32$ & $3 / 128$ & $45 / 128$ & 0 & $1 / 256$ & $1 / 256$ & $15 / 128$ & 0.0085 & 0.0084 & 0.8633 \\
\hline \multirow[t]{4}{*}{120} & 0.0000 & $1 / 9$ & $1 / 9$ & $1 / 9$ & $1 / 9$ & $1 / 9$ & $1 / 9$ & $1 / 9$ & $1 / 9$ & $1 / 9$ & 0.0084 & 0.0083 & 0.9472 \\
\hline & 0.1562 & $5 / 16$ & $5 / 24$ & $5 / 48$ & $3 / 32$ & $3 / 64$ & $3 / 64$ & $1 / 16$ & $1 / 16$ & $1 / 16$ & 0.0085 & 0.0085 & 0.9460 \\
\hline & 0.6250 & $1 / 4$ & $1 / 24$ & $1 / 24$ & $1 / 24$ & $1 / 4$ & $1 / 24$ & $1 / 24$ & $1 / 24$ & $1 / 4$ & 0.0058 & 0.0057 & 0.9449 \\
\hline & 0.8347 & $15 / 32$ & 0 & $1 / 32$ & $3 / 128$ & $45 / 128$ & 0 & $1 / 256$ & $1 / 256$ & $15 / 128$ & 0.0043 & 0.0042 & 0.9511 \\
\hline \multirow[t]{4}{*}{240} & 0.0000 & $1 / 9$ & $1 / 9$ & $1 / 9$ & $1 / 9$ & $1 / 9$ & $1 / 9$ & $1 / 9$ & $1 / 9$ & $1 / 9$ & 0.0042 & 0.0042 & 0.9494 \\
\hline & 0.1562 & $5 / 16$ & $5 / 24$ & $5 / 48$ & $3 / 32$ & $3 / 64$ & $3 / 64$ & $1 / 16$ & $1 / 16$ & $1 / 16$ & 0.0043 & 0.0043 & 0.9496 \\
\hline & 0.6250 & $1 / 4$ & $1 / 24$ & $1 / 24$ & $1 / 24$ & $1 / 4$ & $1 / 24$ & $1 / 24$ & $1 / 24$ & $1 / 4$ & 0.0029 & 0.0029 & 0.9466 \\
\hline & 0.8347 & $15 / 32$ & 0 & $1 / 32$ & $3 / 128$ & $45 / 128$ & 0 & $1 / 256$ & $1 / 256$ & $15 / 128$ & 0.0021 & 0.0021 & 0.9504 \\
\hline \multirow[t]{4}{*}{480} & 0.0000 & $1 / 9$ & $1 / 9$ & $1 / 9$ & $1 / 9$ & $1 / 9$ & $1 / 9$ & $1 / 9$ & $1 / 9$ & $1 / 9$ & 0.0021 & 0.0021 & 0.9495 \\
\hline & 0.1562 & $5 / 16$ & $5 / 24$ & $5 / 48$ & $3 / 32$ & $3 / 64$ & $3 / 64$ & $1 / 16$ & $1 / 16$ & $1 / 16$ & 0.0021 & 0.0021 & 0:9499 \\
\hline & 0.6250 & $1 / 4$ & $1 / 24$ & $1 / 24$ & $1 / 24$ & $1 / 4$ & $1 / 24$ & $1 / 24$ & $1 / 24$ & $1 / 4$ & 0.0014 & 0.0014 & 0.9486 \\
\hline & 0.8347 & $15 / 32$ & 0 & $1 / 32$ & $3 / 128$ & $45 / 128$ & 0 & $1 / 256$ & $1 / 256$ & $15 / 128$ & 0.0011 & 0.0010 & 0.9504 \\
\hline
\end{tabular}

For $n \geq 60$, the values shown for the exact variance $\nu^{2}(R)$, the asymptotic variance $\operatorname{Var}_{a s}(R)$ of the product-moment correlation coefficient and the coverage probability of the confidence interval $\operatorname{Prob}_{C}(\underline{\rho}, \bar{\rho})$ have been obtained by simulation with 40,000 replications per experiment. $\pi_{i j}=P(X=i, Y=j), 0 \leq i, j \leq 2$ are the joint population genotype frequencies for SNPs $X$ and $Y$.

correlation coefficient of \pm 1 is fairly large, $35.95 \%$ and $14.43 \%$ for $n=15$ and $n=30$, respectively, implying that the modification to the confidence procedure described in the previous section (D2a,b) is comparatively likely to apply. Since this modification obviously goes to the conservative direction, the anticonservatism holding for the same values of $n$ for less extreme observations of $R$ is counterbalanced. For $n \geq 60$, deviations of the actual coverage rate from the nominal confidence level reflect almost exclusively the approximation error inherent in the asymptotic construction of the interval estimation procedure under consideration. Actually, convergence of this error to zero (as $n \rightarrow \infty$ ) is distinctively slower than that of $P\left(\left|R_{n}\right|=1\right)$.
Re-Analysis of Linkage Disequilibrium Data from the IL-4 Receptor Alpha Chain Gene

We illustrate the use of the suggested approach to the assessment of associations between SNPs with a typical example which is taken from a recent study on the contribution of the human IL-4 receptor alpha chain gene IL $4 R$ to the risk of developing an atopic syndrome, as measured by the intermediate phenotype serum IgE levels [25]. For the purpose of illustration, we consider a selection of three SNPs, and the set of $3 \times 3$ contingency. Table $6 \mathrm{a}-\mathrm{c}$ shows the pairwise joint distributions obtained in the subsample of $n=158$ subjects with serum IgE levels $\leq 100$.

For these empirical distributions on $\{0,1,2\} \times$ $\{0,1,2\}$, our $S A S$ macro yields the entries in the matrix of point estimates and 95\% confidence intervals for the product-moment correlation coefficient $\rho(\boldsymbol{\pi})$ shown in table 7. 
Table 6. Contingency tables for genotypes at T1914C, Q551R and $\mathrm{S} 478 \mathrm{P}$ at the $I L 4 R$ gene based on data generated by Franjkovic et al. [25]

\begin{tabular}{llll}
\multicolumn{1}{l}{} & & & \\
\hline & Q551R & & \\
\cline { 2 - 3 } & QQ & QR & RR \\
\hline T1914C & & & \\
TT & 20 & 2 & 0 \\
CT & 62 & 21 & 2 \\
CC & 27 & 21 & 3 \\
\hline
\end{tabular}

b

\begin{tabular}{llll}
\hline S478P & & \\
\cline { 2 - 2 } & SP & PP \\
\hline
\end{tabular}

\begin{tabular}{crrr}
\hline T1914C & & & \\
TT & 20 & 2 & 0 \\
CT & 65 & 19 & 1 \\
CC & 27 & 22 & 2 \\
\hline
\end{tabular}

c

\begin{tabular}{rrrr}
\hline & \multicolumn{1}{l}{ S478P } & \\
\cline { 2 - 4 } & SS & SP & PP \\
\hline Q551R & & - & \\
QQ & 107 & 2 & 0 \\
QR & 5 & 39 & 0 \\
RR & 0 & 2 & 3 \\
\hline
\end{tabular}

Table 7. Point estimates and $95 \%$ confidence intervals in parenthesis computed from the genotype distributions for pairs of SNPs shown in table 6

\begin{tabular}{|c|c|c|c|}
\hline & T1914C & Q551R & S478P \\
\hline T1914C & & $\begin{array}{l}0.2718 \\
(0.1346,0.3988)\end{array}$ & $\begin{array}{l}0.2891 \\
(0.1500,0.4171)\end{array}$ \\
\hline Q551R & & & $\begin{array}{l}0.8984 \\
(0.8130,0.9460)\end{array}$ \\
\hline
\end{tabular}

As regards the confidence limits, the results differ markedly from those obtained by means of the naïve approach where data are treated as if they were taken from a bivariate normal distribution. For the correlation between the third couple of SNPs (Q551R, S478P), the latter
Table 8. Asymptotic efficiency $e^{*}\left(\hat{\rho}_{n}^{(H)}, \hat{\rho}_{2 n}^{(D)}\right)$ under Hardy-Weinberg equilibrium of the haplotype-based relative to the genotypebased estimator of $\rho$ for various configurations of true haplotype frequencies $h_{1}, \ldots h_{4}$

\begin{tabular}{llllll}
\hline$h_{1}$ & $h_{2}$ & $h_{3}$ & $h_{4}$ & $\rho$ & $e^{*} \hat{\rho}_{n}^{(H)}, \hat{\rho}_{2 n}^{(D)}$ \\
\hline 0.075 & 0.225 & 0.175 & 0.525 & 0.000 & 2.0000 \\
0.500 & $0.16 \overline{6}$ & $0.16 \overline{6}$ & $0.16 \overline{6}$ & 0.250 & 1.8621 \\
0.400 & 0.100 & 0.100 & 0.400 & 0.600 & 1.6400 \\
0.375 & 0.250 & 0.000 & 0.375 & 0.600 & 1.1666 \\
0.450 & 0.050 & 0.050 & 0.450 & 0.800 & 1.3600 \\
\hline
\end{tabular}

produces the $95 \%$ confidence interval $(0.8638,0.9246)$ being considerably shorter than the asymptotically correct one. However, this apparent gain in precision of the interval estimate is bought at the price of a dramatic increase of the risk of non-coverage: A simulation experiment with 40,000 samples of size $n=158$ from the bivariate distribution defined by the entries in table $6 c$, gave a coverage probability of 0.6391 for the naïve interval estimation procedure at nominal level $1-\alpha=0.95$.

\section{Comparison of the Efficiency of the Genotype-Based \\ Estimator and the Haplotype-Based Estimator}

Table 8 shows the results for comparing the efficiency of the genotype-based estimator with that of the haplotype-based estimator. The results are displayed for a selection of haplotype distributions for which the true value of $\rho$ ranges from 0 to 0.80 . As becomes obvious from these values, the gain in efficiency entailed by making use of haplotype frequency data depends on the true value of $\rho$ and is worth of taking the risk of erroneously assuming HWE only for low to moderate correlations. Doubling the sample size yields double asymptotic efficiency of the estimator of $\rho$ only in the extreme case that both gene loci involved are independent. Furthermore, it has to be taken into account that the relative efficiencies shown in the table have been computed under the assumption that the haplotype frequencies $h_{1}, \ldots, h_{4}$ can be estimated directly from the data which requires phased genotyping. With unphased genotype data, the natural, fully efficient estimator of the $h_{\nu}(\nu=1, \ldots, 4)$ has to be replaced with a subefficient estimator based on an incomplete-data likelihood [for details, see ref. 29]. Actually, this means that the population haplotype frequencies have to be estimated by functions of the observed relative frequencies from $3 \times 3$ tables like those shown in table 6 . As suggested by the results shown in table 4 , the corresponding estimator 
of $\rho$ has roughly the same standard error as the empirical product-moment correlation coefficient calculated directly from the joint empirical distribution of genotypes.

\section{Discussion}

Measures of LD are important for designing association studies until whole-genome resequencing becomes routinely available [6]. However, there is no natural scale for measuring it; many measures have been proposed for two SNPs, and the most important are $D^{\prime}$ and the Pearson product-moment correlation coefficient $R$ [6]. Although $D^{\prime}$ has a unimodal and symmetric distribution, with the maximum at the disease locus itself [16], and although it is directly related to the recombination fraction under random sampling in a cohort study [see, e.g., ref. 13 ], $D^{\prime}$ can indicate high $\mathrm{LD}$ even when one allele is very rare as illustrated above. $D^{\prime}$ is thus of limited use for applications.

In contrast, $R^{2}$ reflects statistical power to detect $\mathrm{LD}$ [6]: with $n R^{2}$ being the Pearson test statistic for independence in a $2 \times 2$ contingency table of haplotype counts, a low absolute value of $R$ corresponds to a large sample size, $n$, that is required to detect the LD between the markers. Indeed, under specific assumptions for the underlying genetic model, a direct functional relationship between the power of a study and the empirical correlation coefficient $R$ between the typed and the causal SNP can be established. However, in current applications, the empirical Pearson product-moment correlation coefficient $R$ based on haplotypes is commonly employed rather than the one based on genotype data. The resulting theoretical correlation coefficients are identical in case of HWE, and the genotype-based estimator may play the same role as the haplotype-based estimator in these situations, e.g., for power calculations, the selection of tagSNPs, and so on. An extension to the case where HWE is not fulfilled needs to be addressed in a separate paper. However, we want to stress that if HWE is violated, haplotyping introduces bias into the estimates as shown above (table 4). In addition, the adequacy of phase determination heavily relies on the LD between SNPs, and estimates are generally imprecise with moderate to low LD [see, e.g., ref. 30].

To be sure, calculating product-moment correlation coefficients is not the only possibility of estimating the correlation between counting variables. In standard statistical data analysis, rank-correlation methods are wide- ly recommended for assessing the association between quantitative variables whose joint distribution exhibits marked deviations of whatever kind from the bivariate Gaussian form [see, e.g., ref. 31, p. 397]. However, when considering allele counts from SNP-based genotyping, there are at least two reasons why this recommendation seems to be problematic:

(i) Counting identifiable objects is a process which obviously leads to a unique result leaving no room for applying non-trivial one-to-one transformations of whatever mathematical form. In the terminology of measurement theory [see, e.g., ref. 32], this means that such values are measured on an absolute scale so that there is no reason to replace them with their ranks in a first step preliminary to any kind of further statistical analysis.

(ii) When primary interest is in measuring the degree of association between the variables rather than testing for independence, even the most popular correlation measures based on ranks are of limited if any use. In fact, neither Spearman's Rho nor Kendall's Tau, although frequently considered as biased estimators of the productmoment correlation [see, e.g., ref. 33, p. 155], has a population counterpart suggesting itself to intuition as a plausible measure of association. Specifically, Tau estimates the difference between the probability of a con- and a discordance with respect to the sign of the differences obtained in both components within two pairs $\left(X_{1}, Y_{1}\right)$, $\left(X_{2}, Y_{2}\right)$. Formally speaking, the population analogue of Tau is $\tau=P\left(\left(X_{1}-X_{2}\right)\left(Y_{1}-Y_{2}\right)>0\right)-P\left(\left(X_{1}-X_{2}\right)\left(Y_{1}-Y_{2}\right)\right.$ $<0)$, and this quantity will not easily be recognized by a statistical practitioner as a surrogate for the population product-moment correlation $\rho$. A thorough study of the basic properties of the rank correlation coefficient [as has been carried out in the classical work of 34 and 35] reveals that the use of Rho as an association measure is still more difficult to motivate. Instead of estimating a single functional on the space of all bivariate distributions, Rho turns out to be a weighted sum of Tau and an unbiased estimator of the so-called grade correlation. The definition of the latter is in terms of triplet - rather than pairwise con- and discordance probabilities and reads $\kappa \equiv 3\left(P\left(\left(X_{1}-X_{2}\right)\left(Y_{1}-Y_{3}\right)>0\right)-P\left(\left(X_{1}-X_{2}\right)\right.\right.$ $\left.\left.\left(Y_{1}-Y_{3}\right)<0\right)\right)$.

Another apparently natural candidate for the choice of a suitable measure of association between ordered categories is the polychoric correlation $\rho_{P C}$. Like several other statistical concepts enjoying considerable popularity within the social sciences, the notion of polychoric correlation presupposes a fully specified latent variable mod- 
Table 9. Genotype frequencies corresponding to haplotype frequencies as given in the counterexample demonstrating noninvariance of the polychoric correlation under the assumption of Hardy-Weinberg equilibrium

\begin{tabular}{llll}
\hline$X$ & $Y$ & & \\
\cline { 2 - 4 } & 0 & 1 & 2 \\
\hline 0 & 0.16 & 0.08 & 0.01 \\
1 & 0.08 & 0.34 & 0.08 \\
2 & 0.01 & 0.08 & 0.16 \\
\hline
\end{tabular}

el [see, e.g., ref. 36, ch. 12]. Accordingly, the inferential procedures which have been developed for $\rho_{P C}$ [for a concise overview, see ref. 37], are valid only under strong parametric assumptions inaccessible to direct model checking by means of the observed data. Another disadvantage of the polychoric correlation is that it lacks the invariance property established in this paper for the product-moment correlation. Actually, we give a simple numerical counterexample in Appendix III showing that the polychoric correlation fails to remain invariant under replacing haplotype with HWE-derived genotype frequencies.

We conclude that the reconstruction of haplotypes in a first step and estimation in a second is not required for estimating the degree of LD between SNPs. Instead, we recommend estimation of the Pearson product-moment correlation coefficient $R$ as measure of $\mathrm{LD}$, and $R$ should be estimated directly from genotype data.

\section{Acknowledgements}

The first author would like to thank Gunter Schumann (Institute of Psychiatry, King's College London) for drawing his attention to the problem treated in this paper. The work of Andreas Ziegler was supported by the Deutsche Forschungsgemeinschaft and the Bundesministerium für Bildung und Forschung. Both authors gratefully acknowledge two anonymous referees for very helpful and constructive suggestions for improving an initial version of this paper.

\section{Appendix I: Derivation of the Asymptotic Variance of the Product-Moment Correlation between Counting Variables}

For convenience, the collection $\pi$ of multinomial parameters is assumed to have the form of a column vector of length $(K+1)^{2}$ obtained by concatenating the rows of the corresponding $(K+1)$ $\times(K+1)$ matrix in the natural way. As explained in the section on the asymptotic distribution of the empirical product-moment correlation, the population correlation coefficient under estimation is a differentiable function of the argument $\boldsymbol{\pi}$. The partial derivative of $\rho(\pi)$ with respect to any of the components of the parameter vector $\pi$ admits the representation

$$
\begin{aligned}
& \frac{\partial}{\partial \pi_{i j}} \rho(\boldsymbol{\pi})= \\
& \frac{i j-i e_{2}(\boldsymbol{\pi})-j e_{1}(\boldsymbol{\pi})}{v_{1}^{1 / 2}(\boldsymbol{\pi}) v_{2}^{1 / 2}(\boldsymbol{\pi})}-\frac{1}{2} \rho(\boldsymbol{\pi})\left[\frac{i\left(i-2 e_{1}(\boldsymbol{\pi})\right)}{v_{1}(\boldsymbol{\pi})}+\frac{j\left(j-2 e_{2}(\boldsymbol{\pi})\right)}{v_{2}(\boldsymbol{\pi})}\right],
\end{aligned}
$$

being valid for any $\pi$ such that none of the marginal variances $v_{1}(\boldsymbol{\pi}), v_{2}(\boldsymbol{\pi})$ vanishes. Furthermore, the covariance matrix, say $\Sigma_{\pi}$, of the normalized vector $\sqrt{n}(\hat{\pi}-\pi)$ of observed relative frequencies is well known to be given by

$$
\Sigma_{\pi} D_{\pi}-\pi \pi^{\prime}
$$

with

$$
\boldsymbol{D}_{\pi}=\left(\begin{array}{cccccc}
\pi_{00} & 0 & \cdots & \cdots & \cdots & 0 \\
0 & \pi_{01} & 0 & \cdots & \cdots & 0 \\
0 & 0 & \pi_{02} & \cdots & \cdots & 0 \\
\vdots & \vdots & \vdots & \ddots & \vdots & \vdots \\
0 & 0 & \cdots & \cdots & \pi_{K, K-1} & 0 \\
0 & 0 & \cdots & \cdots & 0 & \pi_{K K}
\end{array}\right)
$$

Finally, it is an elementary exercise to show that the quadratic form associated with the multinomial covariance matrix (10) admits the representation

$$
\zeta^{\prime} \Sigma_{\pi} \zeta=\sum_{i=0}^{K} \sum_{j=0}^{K} \zeta_{i j}^{2} \pi_{i j}-\left(\sum_{i=0}^{K} \sum_{j=0}^{K} \zeta_{i j} \pi_{i j}\right)^{2}
$$

at any $\zeta=\left(\zeta_{00}, \ldots, \zeta_{0 K}, \ldots, \zeta_{K 0}, \ldots, \zeta_{K K}\right) \in \mathbb{R}^{(K+1)^{2}}[38$, eq. 2.4]. Formula (5) follows, then, by substituting in (11) each $\zeta_{i j}$ by the expression on the right-hand side of Equation (9).

\section{Appendix II: Proof That Independence Implies $\operatorname{Var}_{\text {as }}(R)=1 / n$}

For $\rho^{2}(\boldsymbol{\pi})=0$, equation (5) can be simplified to

$$
\begin{aligned}
& n \operatorname{Var}_{a s}(R) \frac{1}{v_{1}(\boldsymbol{\pi}) v_{2}(\boldsymbol{\pi})} \\
& \left\{\sum_{i=0}^{K} \sum_{j=0}^{K}\left[i j-i e_{2}(\boldsymbol{\pi})-j e_{1}(\boldsymbol{\pi})\right]^{2} \pi_{i j}-\left(\sum_{i=0}^{K} \sum_{j=0}^{K}\left[i j-i e_{2}(\boldsymbol{\pi})-j e_{1}(\boldsymbol{\pi})\right] \pi_{i j}\right)^{2}\right\} \\
& =\frac{E[X Y-X E(Y)-Y E(X)]^{2}-(E[X Y-X E(Y)-Y E(X)])^{2}}{\operatorname{Var}(X) \operatorname{Var}(Y)} .
\end{aligned}
$$


Since $X$ has been assumed to be independent of $Y$, we have

$$
E[X Y-X E(Y)-Y E(X)]=-E(X) E(Y),
$$

and

$$
\begin{aligned}
& \operatorname{Var}(X) \operatorname{Var}(Y)=E[(X-E(X))(\mathrm{Y}-\mathrm{E}(Y))]^{2} \\
& =E[(X Y-E(X) Y-E(X) Y)+E(X) E(Y)]^{2} \\
& =E[X Y-E(X) Y-E(X) Y]^{2} \\
& +2 E(X) E(Y) E[X Y-E(X) Y-E(X) Y]+E^{2}(X) E^{2}(Y) \\
& =E[X Y-X E(Y)-Y E(X)]^{2} \\
& +2 E(X) E(Y) \cdot[-E(X) E(Y)]+E^{2}(X) E^{2}(Y) \\
& =E[X Y-X E(Y)-Y E(X)]^{2}-E^{2}(X) E^{2}(Y) .
\end{aligned}
$$

Plugging in (13) in (14) yields the identity

$$
\begin{aligned}
& E[X Y-X E(Y)-Y E(X)]^{2}-(E[X Y-X E(Y)-Y E(X)])^{2} \\
& =\operatorname{Var}(X) \operatorname{Var}(Y),
\end{aligned}
$$

which in view of (9) implies $n \operatorname{Var}_{a s}(R)=1$.

\section{Appendix III: Counterexample Demonstrating Noninvariance of the Polychoric Correlation under Replacing Haplotype with Genotype Frequencies in Presence of Hardy-Weinberg Equilibrium}

Let the population haplotype frequencies have values $h_{1}=$ $0.40, h_{2}=0.10, h_{3}=0.10, h_{4}=0.40$. Plugging-in these values in the entries in table 2 leads to the joint genotype distribution shown in table 9. From the latter, the polychoric correlation coefficient is computed to be (e.g., by means of the $S A S$ procedure FREQ) $\rho_{P C}^{G E N O}=0.7180$. In contrast, computing the polychoric correlation from the corresponding haplotype distribution yields the value $\rho_{P C}^{H A P}=0.8090$ exceeding distinctly the value of $\rho_{P C}^{G E N O}$.

\section{Electronic Database Information}

The software developed for estimating the empirical correlation coefficient and the corresponding confidence intervals can be retrieved from http://zihost5.zi-mannheim.de/SNP_CORR/ corr_count.sas.

\section{References}

1 Ziegler A, König IR: A statistical Approach to Genetic Epidemiology: Concepts and Applications. Weinheim, Wiley-VCH, 2006.

-2 Helgadottir A, Thorleifsson G, Manolescu A, Gretarsdottir S, Blondal T, Jonasdottir A, Jonasdottir A, Sigurdsson A, Baker A, Palsson A, Masson G, Gudbjartsson D, Magnusson KP, Andersen K, Levey AI, Backman VM, Matthiasdottir S, Jonsdottir T, Palsson S, Einarsdottir H, Gunnarsdottir S, Gylfason A, Vaccarino V, Hooper VC, Reilly MP, Granger CB, Austin H, Rader DJ, Shah SH, Quyyumi AA, Gulcher JR, Thorgeirsson G, Thorsteinsdottir U, Kong A, Stefansson K: A common variant on chromosome 9p21 affects the risk of myocardial infarction. Science 2007;316:1491-1493.

-3 McPherson R, Pertsemlidis A, Kavaslar N, Stewart A, Roberts R, Cox DR, Hinds DA, Pennacchio LA, Tybjaerg-Hansen L, Folsom AR, Boerwinkle E, Hobbs HH, Cohen JC: A common allele on chromosome 9 associated with coronary heart disease. Science 2007; 316:1488-1491.

4 Samani NJ, Erdmann J, Hall AS, Hengstenberg C, Mangino M, Mayer B, Dixon RJ, Meitinger T, Braund P, Wichmann H-E, Barrett JH, König IR, Stevens S, Szymczak S, Tregouet D-A, Iles MM, Pahlke F, Pollard H, Lieb W, Cambien F, Fischer M, Ouwehand W, Balmforth AJ, Baessler A, Ball SG, Strom TM, Brænne I, Gieger C, Deloukas P, Tobin MD, Ziegler A, Thompson JR, Schunkert H, The Wellcome Trust Case Control Consortium, and The Cardiogenics Consortium. Genome-wide association analysis of coronary artery disease. N Engl J Med 2007;357: 443-453.
5 The Wellcome Trust Case Control Consortium (WTCCC). Genome-wide association study of 14,000 cases of seven common diseases and 3,000 shared controls. Nature 2007;60:661-678.

6 Balding DJ: A tutorial on statistical methods for population association studies. Nat Rev Genet 2006;7:781-791.

7 Garner C, Slatkin M: On selecting markers for association studies: patterns of linkage disequilibrium between two and three diallelic loci. Genet Epidemiol 2003;24:57-67.

8 Stram DO: Tag SNP selection for association studies. Genet Epidemiol 2004;27:365-374.

9 Wang T, Zhu X, Elston RC: Improving power in contrasting linkage-disequilibrium patterns between cases and controls. Am J Hum Genet 2007;80:911-920.

10 Zaykin DV, Meng Z, Ehm MG: Contrasting linkage disequilibrium patterns between cases and controls as a novel associationmapping method. Am J Hum Genet 2006;78: 737-746

11 Conrad DF, Jakobsson M, Coop G, Wen X, Wall JD, Rosenberg NA, Pritchard JK: A worldwide survey of haplotype variation and linkage disequilibrium in the human genome. Nat Genet 2006;38:1251-1260.

12 Weiss KM, Clark AG: Linkage disequilibrium and the mapping of complex human traits. Trends Genet 2002;18:19-24.

13 Morton NE, Zhang W, Taillon-Miller P, Ennis S, Kwok P-Y, Collins A: The optimal measure of allelic association. Proc Natl Acad Sci USA 2001;98:5217-5221.

14 Hedrick PW: Gametic disequilibrium measures: Proceed with caution. Genetics 1987; 117:331-341.
15 Lewontin R: On measures of gametic disequilibrium. Genetics 1998;120:849-852.

16 Devlin B, Risch N: A comparison of linkage disequilibrium measures for fine-scale mapping. Genomics 1995;29:311-322.

17 Lewontin R: The interaction of selection and linkage. I. General considerations: Heterotic models. Genetics 1964;49:49-67.

18 Hill WG, Robertson A: Lineage disequilibrium in finite populations. Theor Appl Genet 1968;38:226-231.

19 Langley CH, Tobari YN, Kojima K-I: Linkage equilibrium in natural populations of Drosophila melanogaster. Genetics 1974;78: 921-936.

20 Weir BS: Inferences about linkage disequilibrium. Biometrics 1979;35:235-254.

21 Thomas DC: Statistical Methods in Genetic Epidemiology. New York, Oxford University Press, 2004.

22 Bishop YMM, Fienberg SE, Holland PW: Discrete Multivariate Analysis: Theory and Practice. Cambridge (MA), MIT Press, 1975.

23 Brown MB, Benedetti JK: Sampling behavior of tests of correlation in two-way contingency tables. J Am Stat Assoc 1977;72:309-315.

24 Duncan GT, Layard MWJ: A Monte Carlo study of asymptotically robust tests for correlation coefficients. Biometrika 1973;60: 551-558.

25 Franjkovic I, Gessner A, König IR, Kissel K, Bohnert A, Hartung A, Ohly A, Ziegler A, Hackstein H, Bein G: Effects of common atopy-associated amino acid substitutions in the IL-4 receptor alpha chain on IL-4 induced phenotypes. Immunogenet 2005;56: 808-817. 
26 Sasieni PD: From genotypes to genes: doubling the sample size. Biometrics 1997;53: 1253-1261.

27 Wellek S: Tests for establishing compatibility of an observed genotype distribution with Hardy Weinberg equilibrium in the case of a biallelic locus. Biometrics 2004;60:694-703.

28 Casella G, Berger RL: Statistical Inference. Pacific Grove (CA), Duxbury, ed 2, 2002.

29 Excoffier L, Slatkin M: Maximum-likelihood estimation of molecular haplotype frequencies in a diploid population. Mol Biol Evol 1995;12:921-927.
30 Beckmann L, Ziegler A, Duggal P, BaileyWilson JE: Haplotypes and haplotype-tagging single-nucleotide polymorphism: Presentation group 8 of Genetic Analysis Workshop 14. Genet Epidemiol 2005;29 (Suppl 1):S59-S71.

31 SPSS. SPSS 13.0 Base User's Guide. New York, Prentice Hall, 2004.

32 Roberts FS: Measurement Theory - With Applications to Decision Making, Utility, and the Social Sciences. Reading (MA), Addison-Wesley, 1979.

33 S-PLUS. S-PLUS 6.0 Guide to Statistics, vol 1. Seattle (WA), Data Analysis Division, MathSoft, 2000.

34 Hoeffding W: A class of statistics with asymptotically normal distribution. Ann Math Stat 1949;19:293-326.
35 Kruskal WH: Ordinal measures of association. J Am Stat Assoc 1958;53:814-861.

36 Krzanowski WJ, Marriott FHC: Multivariate Analysis, Part 2: Classification, Covariance Structures and Repeated Measurements. London, Arnold, 1995.

37 Drasgow F: Polychoric and polyserial correlations; in Kotz S, Johnson NL, Read CB (eds): Encyclopedia of Statistical Sciences, vol 7, pp 68-74. New York, Wiley, 1986.

38 Goodman LA, Kruskal WH: Measures of association for cross classifications, IV: Simplification of asymptotic variances. J Am Stat Assoc 1972;67:415-421. 